\title{
FDTD Computational Study of Ultra-Narrow TM Non-Paraxial Spatial Soliton Interactions
}

\author{
Zachary Lubin, Jethro H. Greene, and Allen Taflove, Fellow, IEEE
}

\begin{abstract}
We consider the interaction between two $(1+1) D$ ultra-narrow optical spatial solitons in a nonlinear dispersive medium using the finite-difference time-domain (FDTD) method for the transverse magnetic (TM) polarization. The model uses the general vector auxiliary differential equation (GVADE) approach to include multiple electric-field components, a Kerr nonlinearity, and multiple-pole Lorentz and Raman dispersive terms. This study is believed to be the first considering narrow soliton interaction dynamics for the TM case using the GVADE FDTD method, and our findings demonstrate the utility of GVADE simulation in the design of soliton-based optical switches.
\end{abstract}

Index Terms-Finite-difference time-domain method, FDTD, GVADE, nonlinear optics, spatial solitons.

$\mathbf{S}$ PATIAL optical solitons are self-trapped optical beams balancing diffraction and self-focusing due to intensity-induced modifications in the local refractive index. One fascinating feature of solitons is their deflection behavior when in the vicinity of other solitons. This can be exploited for applications in optical routing and guiding or in switching applications in all optical-based interconnects and nanocircuits (for example, [1]).

The work by Aitchison, et al. first reported experimental observations that solitons either repel or attract each other with a periodic evolution over propagation, depending on the relative phase between them [2]. Subsequent studies extended the findings and explored applications; slight variation on the launch angle and relative phase was found to cause a soliton pair to merge into one of the original trajectories [3]. More recent efforts considered interactions in semiconductor media [4], incoherent interactions [5], all-optical switching [6], long-range interactions [7], and the dynamics of interacting, self-focusing beams [8].

An effective numerical technique known as the beam propagation method (BPM) can be used to model soliton interaction. It is a Fourier-based algorithm that solves the nonlinear Schrodinger equation (NLSE) for the envelope of the field. It typically requires low memory for computer implementation. Some limitations, however, are that it makes a scalar approximation, relies on paraxiality, and also depends on slowly-varying envelope conditions for validity without proper modifications

Manuscript received July 10, 2010; revised January 04, 2011; accepted February 25, 2011. Date of publication March 28, 2011; date of current version May $11,2011$.

The authors are with Northwestern University, Evanston, IL 60208 USA (e-mail: z-lubin@northwestern.edu).

Color versions of one or more of the figures in this paper are available online at http://ieeexplore.ieee.org.

Digital Object Identifier 10.1109/LMWC.2011.2126019
[9]. Such modifications to enhance its capability have been proposed in [10], [11].

The finite-difference time-domain (FDTD) method has been previously applied to analyzing problems in nonlinear optics, including solitons [9], [12]-[17]. FDTD accounts for the full-vector nature of the fields, and can readily accommodate inhomogeneous and dispersive media. FDTD does not rely on any simplifying assumptions such as paraxial or scalar approximations as is often employed in a typical analysis with the NLSE. Such assumptions are not appropriate when considering soliton beamwidths on the order of a wavelength. Recently, a new FDTD algorithm was described, which can accommodate more than one electric-field component in media possessing both instantaneous and dispersive nonlinearities, as well as linear material dispersion. Known as the general vector auxiliary differential equation (GVADE) method [15], it has been applied to the study of soliton interactions with nanoscale air gaps embedded in glass [16]. Ultra-narrow solitons involve significant interactions between both longitudinal and transverse electric-field components [14] and the GVADE method accounts for this physics.

In this study, we consider the problem of modeling interacting spatial solitons with beamwidths on the order of one wavelength using the GVADE FDTD method. The separation of the solitons as well as their relative phase is varied, and their influences on the propagation dynamics are qualitatively assessed. Soliton interaction has previously been modeled using FDTD, but only for the transverse electric (TE) polarization case with a single electric-field component, and the model did not incorporate dispersion [13]. To the best of our knowledge, this is the first work utilizing the GVADE FDTD method to simulate ultra-narrow soliton interactions in the transverse magnetic (TM) polarization. Our study is of interest to efforts involved with time-domain numerical techniques for electromagnetic fields, and relevant to both optical switching applications and control of microwave devices given increasing integration of microwave and optical technology.

\section{GVADE FDTD MODEL}

The GVADE method allows for multiple electric-field components to be included in the FDTD domain where a media nonlinearity is present. It also permits integration of linear and nonlinear dispersive models. GVADE has been shown to converge to previously published FDTD results, as well as reproduce known characteristics of higher-order solitons [15]. GVADE could also easily accommodate complex boundary conditions where a variety of materials exist in the propagation path, such as plasmonic nanoscale metals. 


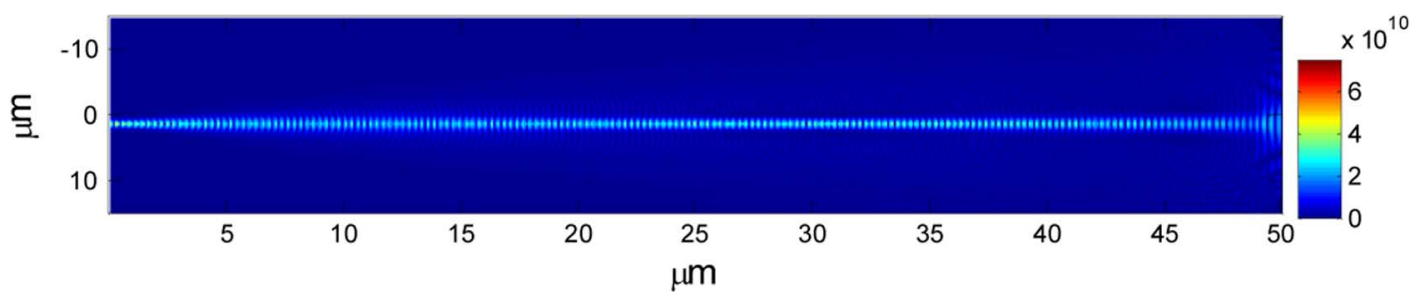

Fig. 1. The magnitude of the electric-field $|\mathrm{E}|$ is plotted from a simulation of a single, ultra-narrow soliton using the GVADE FDTD model.

We consider the transverse magnetic (TM) polarization, modeling two electric-field components $\left(\mathrm{E}_{\mathrm{x}}, \mathrm{E}_{\mathrm{y}}\right)$ and a magneticfield component $\left(\mathrm{H}_{\mathrm{z}}\right)$. We assume fused silica as the propagation medium, having an instantaneous Kerr nonlinearity and a Raman dispersive nonlinearity. A Lorentzian linear dispersion is included, based on a three-pole Sellmeier model as

$$
n^{2}=1+\sum_{i=1}^{3} b_{i} \frac{\lambda^{2}}{\lambda^{2}-\lambda_{i}^{2}}
$$

The model parameters used for all dispersions are the same as those used in [17]. The resonant strengths in (1) are $\mathrm{b}_{1}=0.6961663, \mathrm{~b}_{2}=0.4079426$, and $\mathrm{b}_{3}=0.8974794$, and the resonant wavelengths are $\lambda_{1}=0.0684043 \mu \mathrm{m}$, $\lambda_{2}=0.1162414 \mu \mathrm{m}$, and $\lambda_{3}=9.896161 \mu \mathrm{m}$. For the Raman nonlinear dispersion, the time constants are $\tau_{1}=12.2 \mathrm{fs}$ and $\tau_{2}=32.0 \mathrm{fs}$ with a relative Kerr strength factor of 0.7 .

The computational grid dimensions are $50 \mu \mathrm{m}$ in the $\mathrm{x}$-(propagation) dimension and $30 \mu \mathrm{m}$ in the $\mathrm{y}$-(transverse) dimension. No outer absorbing boundary is used but the grid is sufficiently extended to avoid reflections over the time span of interest. The grid cell-size is $\Delta=10 \mathrm{~nm}$ and the time-step increment is $\tau=4.17 \times 10^{-18} \mathrm{sec}$. An excitation waveform is generated at the far-left side of the computational grid. The excitation is applied to the $\mathrm{H}_{\mathrm{z}}$ component as

$$
\begin{aligned}
\mathrm{H}_{z}=A_{o}[\sin (\omega \mathrm{t}) \operatorname{sech} & \left(\frac{\mathrm{y}-\mathrm{y}_{1}}{\mathrm{w}_{\mathrm{o}}}\right) \\
& \left.+c \sin (\omega \mathrm{t}+\theta) \operatorname{sech}\left(\frac{\mathrm{y}-\mathrm{y}_{2}}{\mathrm{w}_{\mathrm{o}}}\right)\right]
\end{aligned}
$$

which is of the form for a pair of fundamental bright spatial solitons [13]. We use a carrier wavelength of $800 \mathrm{~nm}$. The terms $\mathrm{y}_{1}$ and $\mathrm{y}_{2}$ are the locations of the transverse profile peaks, and their separation distance is given by

$$
\mathrm{d}=|\mathrm{y} 2-\mathrm{y} 1|
$$

The relative phase between the solitons is specified by $\theta$ and the amplitude $A_{o}$ is $2.0 \times 10^{8} \mathrm{~A} / \mathrm{m}$. The characteristic width $\mathrm{w}_{o}$ is $261 \mathrm{~nm}$ and gives a full-width at half-maximum to the dielectric wavelength ratio of $\mathrm{FWHM} / \lambda_{\mathrm{d}}=1.25$. With a beamwidth on the order of a wavelength, the soliton is ultra-narrow and highly non-paraxial. The GVADE FDTD method is well-suited to model such optics, as both electric-field components are accounted for through solution of the vector Maxwell's equations. The constant $c$ is set to unity when a soliton pair is desired, and to zero in the single soliton case. The latter is shown in Fig. 1, a false-color plot of the magnitude of the electric-field $|\mathrm{E}|$ in the (a)

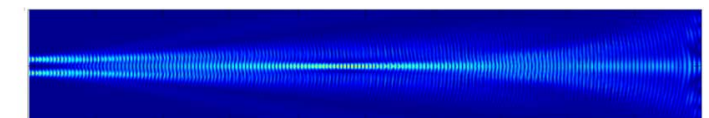

(b)

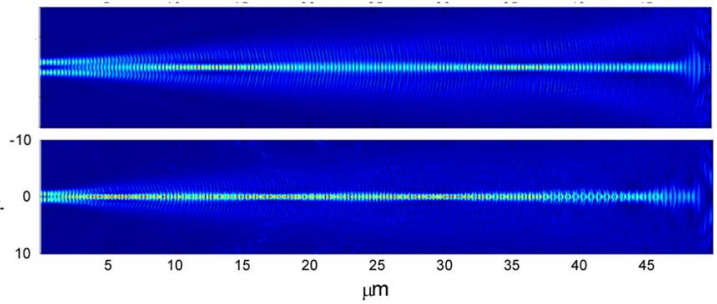

Fig. 2. $|\mathrm{E}|$ for two solitons launched in phase, parallel to each other, with $d_{n}$ of (a) 3.5 ; (b) 2.5 ; (c) 1.5 . The solitons are seen to merge into a single beam, and the onset of their merge depends on the initial pair separation. The color scale is the same as shown in Fig. 1.

FDTD grid at 60000 time-steps. The transverse profile is observed to remain preserved over a propagation length exceeding 120 diffraction lengths, where a diffraction length (or Rayleigh range) is defined as $\mathrm{L}_{\mathrm{d}}=\pi \mathrm{w}_{\mathrm{o}}^{2} / \lambda_{\mathrm{d}}$. These results were obtained using an Intel Xeon 3.2 GHz 20-processor Linux cluster and typical simulation time was 25 hours. Physical memory usage was $134 \mathrm{MB}$ per process.

\section{SOLITON INTERACTION EXPERIMENTS}

The GVADE FDTD model described above is used to simulate a pair of ultra-narrow solitons with profiles as given in (2), launched in parallel with a spatial separation of $d$ as given in (3). The relative phase $\theta$ between the solitons is set to zero. The spacing $d$ is varied in terms of the FWHM; define a "normalized" soliton separation as $d_{n}=d / F W H M$. Fig. 2 shows results for the cases of $d_{n}=3.5,2.5$, and 1.5. We have observed what starts as an independent propagation of the soliton pair, but rapidly transforms via mutual attraction to a single soliton-like beam. It is found to retain this form for the length of the grid, extending more than 120 diffraction lengths. As it is apparent from Fig. 2(a), we find that the distance to the soliton "merging" depends on the initial separation; it begins to set in near $\mathrm{x} \sim 15 \mu \mathrm{m}$ for the case of $\mathrm{d}_{\mathrm{n}}=3.5$, but shrinks to $\mathrm{x} \sim 3 \mu \mathrm{m}$ when $\mathrm{d}_{\mathrm{n}}=1.5$ (Fig. 2(c)). While there are slight periodic expansions and focusing of the fused transverse profile, it never "splits", i.e., where we recover the pair within the grid length of $50 \mu \mathrm{m}$. These results illustrate the interaction dynamics of two close-proximity, non-paraxial TM solitons, and can have application to nonlinear optical circuits involving signal "combiners".

The relative phase $\theta$ is now set equal to the antiphase case, $\pi$ radians. The separation $d_{n}$ is again varied while all other simulation parameters remain unchanged. Fig. 3(a) shows the results 


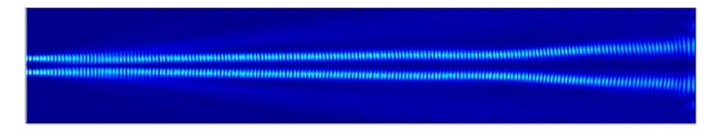

(b)

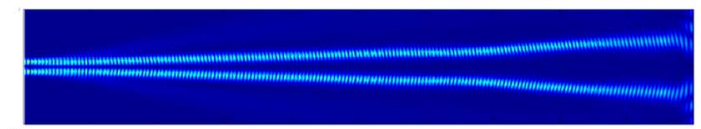

(c)

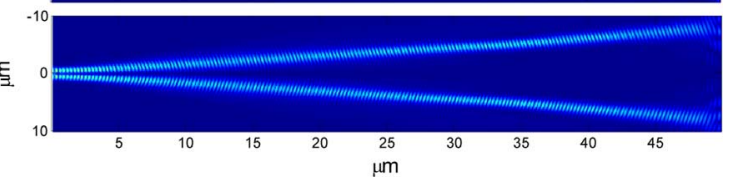

Fig. 3. $|E|$ for two solitons launched antiphase, parallel to each other, with $d_{n}$ of (a) 3.5 ; (b) 2.5 ; (c) 1.5 . An eventual divergence is observed between them, although its onset appears to be heavily influenced by the initial separation distance $d_{n}$. The color scale is the same as shown in Fig. 1.

(a)

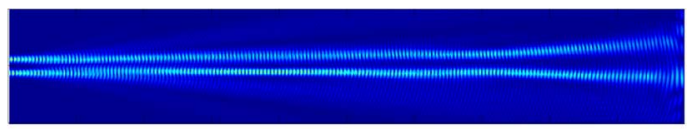

(b)

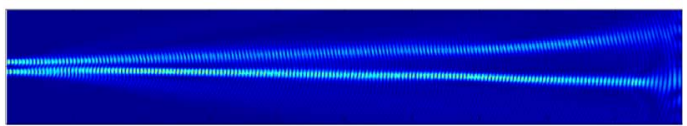

(c)

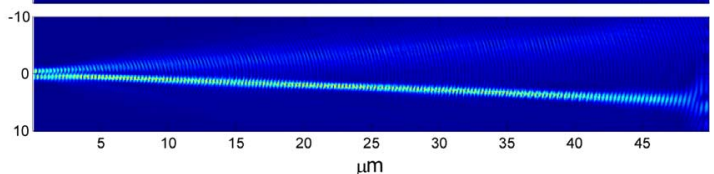

Fig. 4. $|\mathrm{E}|$ for two solitons launched with a relative phase of $\pi / 2$ radians, parallel to each other, with $d_{n}$ of (a) 3.5; (b) 2.5; (c) 1.5. As the spacing $d_{n}$ decreases the lower soliton gains energy at the expense of the other, and starts to take on an oblique trajectory. The color scale is the same as shown in Fig. 1.

for the case of $d_{n}=3.5$. A gradual, mutual repulsion is observed over a propagation distance on the order of $37 \mu \mathrm{m}$. This is followed by a more rapid repulsion extending to the end of the grid. As $d_{n}$ is decreased, the rate of the spatial repulsion grows. As seen in Fig. 3(c), with a separation of $d_{n}=1.5$, the pair begins to repel within a propagation distance of only $3 \mu \mathrm{m}$.

In a third experiment, the phase $\theta$ is set to $\pi / 2$ radians. The results, shown in Fig. 4, exhibit a mutual repulsion between the soliton pair for $\mathrm{d}_{\mathrm{n}}=3.5$ similar to the antiphase case. However, as $d_{n}$ decreases, the lower soliton gains energy at the expense of the other and takes on a large divergence angle with respect to the optical axis. Fig. 4(c) shows the $d_{n}=1.5$ case, where the upper soliton has collapsed due to diffraction and the lower exhibits deflection with increased energy.

The soliton interaction behavior is consistent with the experimental findings of [2], [18] and we believe this to be the first study demonstrating GVADE FDTD numerical techniques applied to this problem.

\section{CONCLUSION}

We have utilized the GVADE FDTD method to study interactions between a pair of TM-polarized, $(1+1) \mathrm{D}$ ultra-narrow spatial solitons, incorporating an instantaneous Kerr nonlinearity and linear and nonlinear dispersions. These results are relevant to switching and control device simulation/design for not only optical wavelengths, but microwave as well as we believe these technologies will find a potential merge in future hybrid wireless. Through new demonstrations, we have shown FDTD as an effective approach to computer-aided design for such applications.

\section{ACKNOWLEDGMENT}

The authors thank Prof. V. Backman, Dr. J. Rogers, Dr. I. Capoglu, J. Spadaro, and N. Borisov for their support.

\section{REFERENCES}

[1] L. S. D. Alcantara, M. A. C. Lima, A. C. Cesar, B. V. Borges, and F. L. Teixeira, "Design of a multifunctional integrated optical isolatorswitch based on nonlinear and nonreciprocal effects," Opt. Eng., vol. 44, pp. 124002.1-124002.9, 2005.

[2] J. S. Aitchison, A. M. Weiner, Y. Silberberg, D. E. Leaird, M. K. Oliver, J. L. Jackel, and P. W. E. Smith, "Experimental observation of spatial soliton interactions," Opt. Lett., vol. 16, pp. 15-17, 1991.

[3] M. Shalaby and A. Barthelemy, "Experimental spatial soliton trapping and switching," Opt. Lett., vol. 16, pp. 1472-1474, 1991.

[4] E. A. Ultanir, G. I. Stegeman, C. H. Lange, and F. Lederer, "Coherent interactions of dissipative spatial solitons," Opt. Lett., vol. 29, pp. 283-285, 2004.

[5] T.-S. Ku, M. Shih, A. A. Sukhorukov, and Y. S. Kivshar, "Coherence controlled soliton interactions," Phys. Rev. Lett., vol. 94, pp. 0639041-063904-4, 2005.

[6] Y. Wu, "New all-optical switch based on the spatial soliton repulsion," Opt. Exp., vol. 14, pp. 4005-4012, 2006.

[7] C. Rotschild, B. Alfassi, O. Cohen, and M. Segev, "Long-range interactions between optical solitons," Nature Phys., vol. 2, pp. 769-774, 2006.

[8] A. A. Ishaaya, T. D. Grow, S. Ghosh, L. T. Vuong, and A. L. Gaeta, "Self-focusing dynamics of coupled optical beams," Phys. Rev. A, vol. 75, pp. 023813-1-023813-5, 2007.

[9] G. Bellanca, R. Semprini, and P. Bassi, "FDTD modeling of spatial soliton propagation,” Opt. Quant. Electron., vol. 29, pp. 233-241, 1997.

[10] T. Fujisawa and M. Koshiba, "Full-vector finite-element beam propagation method for three-dimensional nonlinear optical waveguides," $J$. Lightw. Technol., vol. 20, no. 10, pp. 1876-1884, Oct. 2002.

[11] L. S. D. Alcantara, F. L. Teixeira, A. C. Cesar, and B. V. Borges, "A new full-vectorial FD-BPM scheme: Application to the analysis of magnetooptic and nonlinear saturable media," J. Lightw. Technol., vol. 23, no. 8, pp. 2579-2585, Aug. 2005.

[12] A. Taflove and S. C. Hagness, Computational Electrodynamics: The Finite-Difference Time-Domain Method,3rd ed. Norwood, MA: Artech House, 2005.

[13] R. M. Joseph and A. Taflove, "Spatial soliton deflection mechanism indicated by FD-TD Maxwell's equations modeling," IEEE Photon. Technol. Lett., vol. 6, no. 10, pp. 1251-1254, Oct. 1994.

[14] R. W. Ziolkowski and J. B. Judkins, "Full-wave vector Maxwell equation modeling of the self-focusing of ultrashort optical pulses in a nonlinear Kerr medium exhibiting a finite response time," J. Opt. Soc. Amer. B, vol. 10, pp. 186-198, 1993.

[15] J. H. Greene and A. Taflove, "General vector auxiliary differential equation finite-difference time-domain method for nonlinear optics," Opt. Exp., vol. 14, pp. 8305-8310, 2006.

[16] J. H. Greene and A. Taflove, "Scattering of spatial optical solitons by subwavelength air holes," IEEE Microw. Wireless Comp. Lett., vol. 17, no. 11, pp. 760-762, Nov. 2007.

[17] S. Nakamura, N. Takasawa, and Y. Koyamada, "Comparison between finite-difference time-domain calculation with all parameters of Sellmeier's fitting equation and experimental results for slightly chirped 12 fs laser pulse propagation in a silica fiber," J. Lightw. Technol., vol. 23, no. 2, pp. 855-863, Feb. 2005.

[18] M. Shalaby, F. Reynaud, and A. Barthelemy, "Experimental observation of spatial soliton interactions with a pi/2 relative phase difference," Opt. Lett.., vol. 17, pp. 778-780, 1992. 